\title{
Towards Surface Regularization via Medial Axis Transitions
}

\author{
Frederic F. Leymarie (1), Benjamin B. Kimia (1) and Peter J. Giblin (2) \\ (1) Brown University, Engineering, USA, and (2) Liverpool University, Mathematics, UK. \\ \{leymarie,kimia\}@lems.brown.edu,pjgiblin@liverpool.ac.uk
}

\begin{abstract}
The reconstruction of objects from data in practical applications often leads to surfaces with small perturbations and other artifacts which make the detection of their ridges and generalized axes difficult. We propose an approach to smoothing small structures while preserving ridges which is based on the medial axis structure of the surface. The medial axis of the surface is organized as a graph structure and the closeness of the medial axis graph to points of instability (transitions) is used to identify those structures which are most likely due to perturbations. The removal of these structures is our approach to regularizing both the medial axis and the surface. This paper focuses on a subset of medial transitions arising from protrusions and the method is illustrated for a few synthetic and real images.
\end{abstract}

\section{Introduction}

The need for smoothing surfaces arises in numerous areas. The reconstruction of objects from laser range scanner data, computerized tomography datasets, video or multiple views, among others, often leads to surfaces with relatively small variations from the ideal surfaces. These perturbations interfere with the extraction of abstract-level structure which is needed for further analysis. First, the collection of ridges on an object is a very useful feature-set used in numerous applications. However, the extraction of salient ridges is overwhelmed by the presence of those pertaining to noise or small deformations, e.g., Fig.1.(a). Traditional smoothing methods such as linear filtering [9], mathematical morphology filters [12], or curvature-driven flows [2] smooth small features away but also round off corners and ridges. Anisotropic methods [11,6] were developed to turn smoothing off or reduce the effect of smoothing near discontinuities. However, coarse-scale ridges may not locally be even present at the fine scale, so their detection without smoothing is not possible, a "chicken and egg problem." In addition, even if they are present at fine scales, their strength may not exceed the threshold used in these methods. The rounding of coarsescale discontinuities in smoothing shapes has been recog-

\section{(a)}

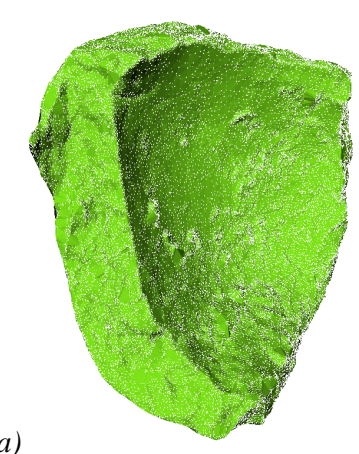

(b)

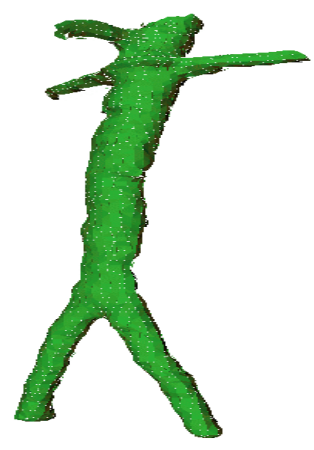

Figure 1: Examples of our automatic surface recovery based on the medial axis [7]: (a) pottery sherd (39,736 point generators); (b) human aorta (7,691 point generators; from [16]).

nized by Pauwels et al. as a significant problem in the use of current smoothing techniques [10]. A second abstract descriptor of surfaces whose computation is affected by noise and small deformations is a generalized cylinder axis useful in numerous applications [16], e.g., Fig.1.(b), where smoothing cannot reveal the sense of small axes "hanging off" larger ones without using an explicit model.

As an alternative to boundary smoothing methods, we consider regularizing the medial axis $(\mathcal{M A})[1,15]$. While this is generally not used to smooth the surface, the regularization of the $\mathcal{M A}$ can implicitly induce a surface regularization method. The majority of the $\mathcal{M A}$ regularization methods assign a measure of salience to a medial point and prune the point when its salience falls below a threshold, either in the detection of the $\mathcal{M A}$ [14], or as a postprocessing step $[13,5]$. This paper proposes a structural smoothing of the $3 \mathrm{D} \mathcal{M A}$ which prunes entire structures based on a notion of transition. In the process it induces a surface regularization method capable of both preserving coarse-scale ridges and highlighting the generalized axes of complex surfaces.

\section{Shock Scaffold and Transitions}

We now review two concepts which are key to our approach, the shock scaffold which identifies a qualitative 
(a)

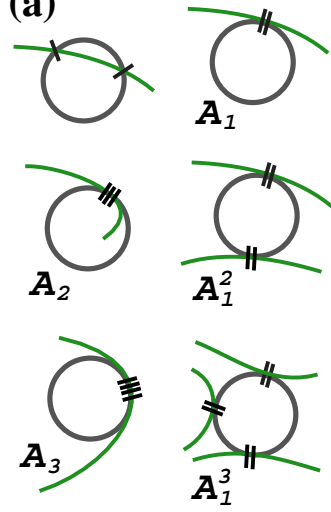

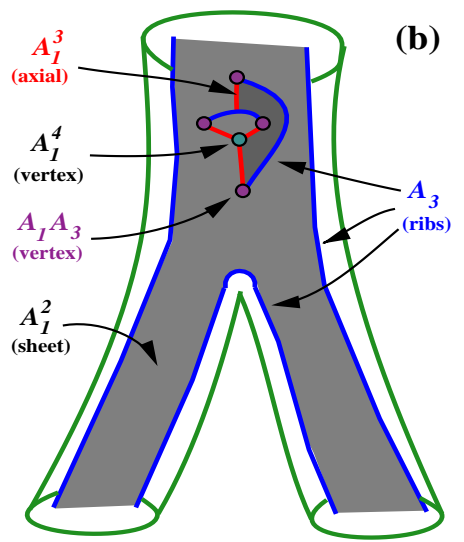

Figure 2: Let $A_{k}^{n}$ denote (a) a circle in 2D or (b) a sphere in 3D osculating a boundary element at $n$ distinct points, each with $k+1$ degree of contact. In 3D there are 5 possible contact types for $\mathcal{M A}$ points: (i) $A_{1}^{2}$ : a sphere with 2 ordinary $A_{1}$ contacts whose center is part of a medial sheet. (ii) $A_{3}$ : limiting case of $2 A_{1}^{2}$ points coming in unison; it corresponds in $3 \mathrm{D}$ to rib curves associated to ridges on the boundary. (iii) $A_{1}^{3}$ : contact sphere with 3 ordinary $A_{1}$ contacts whose center is part of a curve where 3 sheets come together. (iv) $A_{1} A_{3}$ : limiting case of centers of spheres having contact with the surface in 2 places, one near an $A_{1}$ point and one near a $A_{3}$ point; this is where an $A_{1}^{3}$ curve "terminates" together with a $A_{3}$ curve. (v) $A_{1}^{4}$ : the contact sphere has 4 ordinary contacts, and at its center passes $4 A_{1}^{3}$ curves.

structure of the $\mathcal{M A}$, and the notion of transitions which allows for a sequence of discrete transformations of this structure.

The $\mathcal{M A}$ is the closure of the loci of centers of maximal balls tangent to the object surface in two or more points. We have previously proposed a graph structure for the $\mathcal{M A}$ based on a classification of the local form of contact of the ball of tangency leading to five principal types of shock points [3]: $A_{1}^{2}, A_{1}^{3}, A_{3}, A_{1}^{4}$ and $A_{1} A_{3}$, Fig.2. The shock scaffold is a hierarchical structure based on this classification $[7,8]$ : medial sheets are viewed as "hanging off" a scaffold made from medial curves $\left(A_{1}^{3}\right.$ and $\left.A_{3}\right)$ and medial points $\left(A_{1} A_{3}\right.$ and $A_{1}^{4}$ ). The shock scaffold is represented as a directed graph where the medial points are nodes and the medial curves are links in the structure; the medial sheets are then represented by hyperlinks. In this paper, $A_{3}$ and $A_{1}^{3}$ curves are shown in blue and red, respectively. ${ }^{1}$

The second notion required for our proposed approach is one of medial transitions, some of which are well-known in $2 \mathrm{D}$ as the classical instabilities of the $\mathcal{M A}$ : when the shape is slightly perturbed the $\mathcal{M A}$ topology can experi-

\footnotetext{
${ }^{1}$ For color figures, refer to the CD rom for these proceedings or to our website: www.lems.brown.edu/vision/researchAreas/Shocks3D/
}

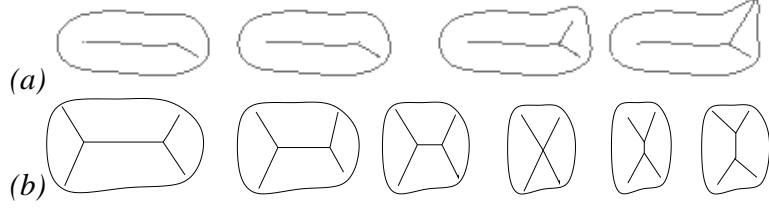

Figure 3: From [4, Fig.1]; (a) The growth of an axis with small perturbations $\left(A_{1} A_{3}\right)$ and $(b)$ the swapping of $\mathcal{M A}$ branches $\left(A_{1}^{4}\right)$ both affect the $\mathcal{M A}$ topology with slight perturbations.

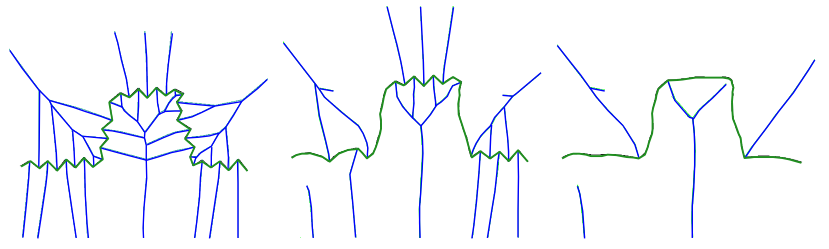

Figure 4: From [15, Fig.1]; the original square wave is perturbed at numerous places. Traditional smoothing methods smooth away both the noise and corners. Instead, the iterative removal of branches of the $\mathcal{M A}$ graph smoothes the shape while preserving its corner (only a subset of the discrete space is shown).

ence large changes, i.e., the growth of an axis, Fig.3.(a), or the swapping of $\mathcal{M A}$ branches, Fig.3.(b). Their complete set can be found in [15]. Fig.4 illustrates how those long branches of the $\mathcal{M A}$ which correspond to a small perturbation of the shape can be removed (the $A_{1} A_{3}$ transition, Fig.3.(a)), thus reversing the effect of the presumed perturbation. This operation can then be iterated, removing noise in the process but retaining the "significant corners" which are seen to have a large distance to the transition point [15].

\section{Regularizing surfaces via Transitions}

A similar process in $3 \mathrm{D}$ requires a formal understanding of the transitions of the $3 \mathrm{D} \mathcal{M A}$ which was recently com-

(a)
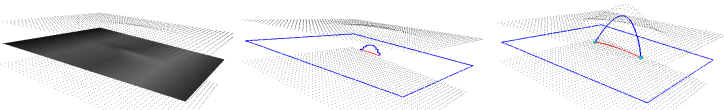

(b)
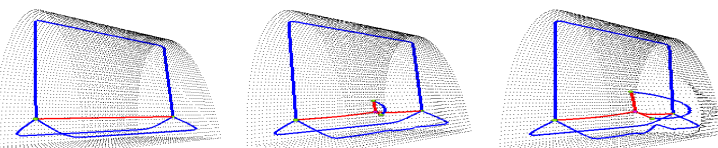

(c)
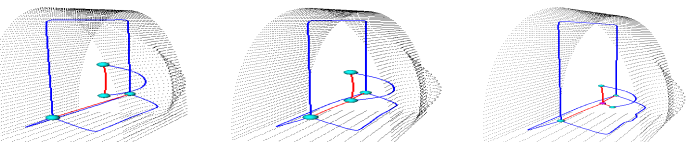

Figure 5: Illustration of 3D transitions [4] due to protrusions, where the shape in each row deforms from left to right passing through a transition in the middle. The transitions are: (a) $A_{1} A_{3}$ I, (b) $A_{1}^{2} A_{3}$-I, and (c) $A_{1}^{2} A_{3}$-II. 
pleted [4]. In this paper we only consider those three transitions which arise from surface protrusions, namely, the $A_{1} A_{3}$-I, $A_{1}^{2} A_{3}$-I and $A_{1}^{2} A_{3}$-II, Fig.5. Our initial datasets are unorganized point generators sampling the surfaces of objects. We now describe the steps required to identify transitions in the shock scaffold graph structure, and perform combined scaffold and surface regularization.

First, we compute the shock scaffold from point generators sampling the surface of an object [7,8]. Second, symmetries pertaining to the interaction of nearby sampled points are used to construct a surface mesh approximating the original object's surface, and the corresponding shocks are removed from the scaffold [7] (Fig.1). Third, in this reduced graph we then identify loop structures defined by an $A_{3}$ rib attached to one or more $A_{1}^{3}$ curves bounding a single $A_{1}^{2}$ medial sheet. Fourth, for a given extracted loop, "walking" along the $A_{1}^{3}$ curves, we identify pairs of contact points on the surface which delimit a protrusion, Fig.6.(c), and permit to build a smooth patch to cut-off the protrusion and close-up the remaining surface Fig.6.(d). Fifth, the collection of extracted loop structures are ordered by their scale, a function of the protrusion's volume as defined by the cut-off patch. This permits a scale-space approach to surface (and scaffold) regularization starting from the smaller protrusions identified by either of the above three transitions. Fig.6.(f-i) show a deformed box where the three transitions arise, are detected and removed.

Fig.7 shows a realistic dataset of the sherd shown in Fig.1.(a). Observe that all three types of transition arise and their removal reveal the salient structures: long $A_{3}$ ribs and $A_{1}^{3}$ axial curves (Fig.7.(b)). The reconstruction in Fig.7.(c) is hard to appreciate in 2D presentations and requires 3D visualization. Fig.8 shows the regularized scaffold on the right which features both regularized ridges and regularized generalized axes, while the regularization of the reconstruction is not possible in a $2 \mathrm{D}$ presentation, it is clear from the scaffold that the significant features like the arms and legs are effectively presented while smoothing the noisy structures. Finally, Fig.9 shows a rather challenging example of a tube-like structure arranged in a knot. The iterative application of transitions reveals the generalized axis as shown in the zoomed box on the right.

\section{Acknowledgements}

We thank the NSF, Grants ITR 0205477 and 0083231 which partially supported this work.

\section{References}

[1] H. Blum. Biological shape and visual science. Journal of Theoretical Biology, 38:205-287, 1973.

[2] M. Gage and R. S. Hamilton. The heat equation shrinking convex plane curves. J. Diff. Geometry, 23:69-96, 1986.
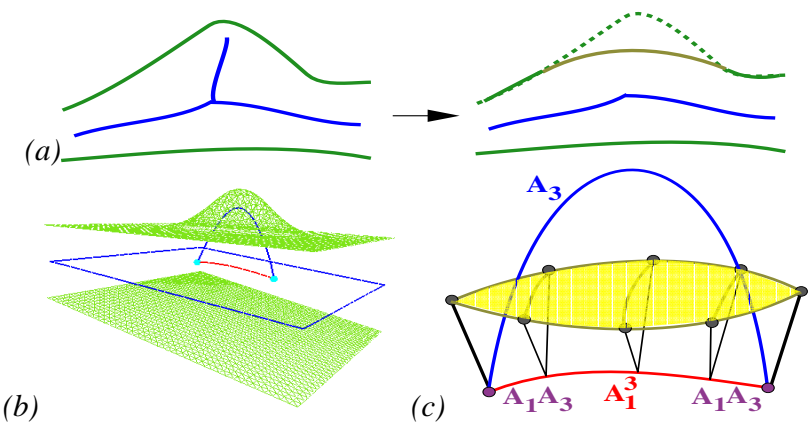

(d)

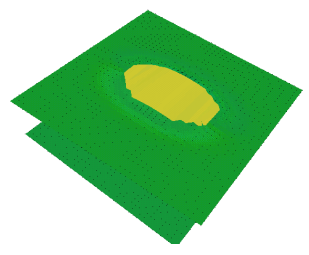

(e)

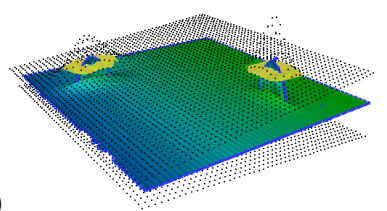

(f)
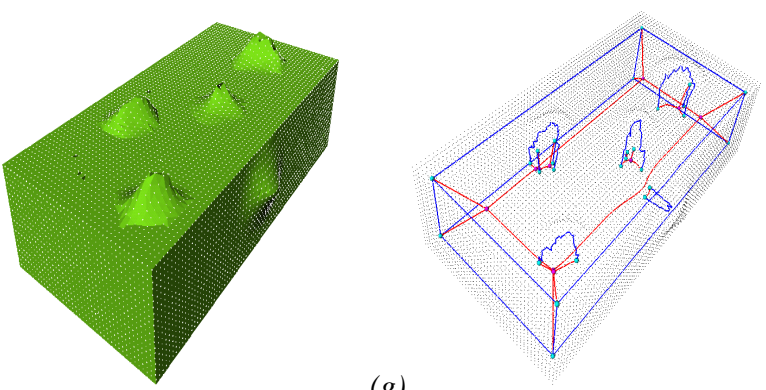

(g)

(h)

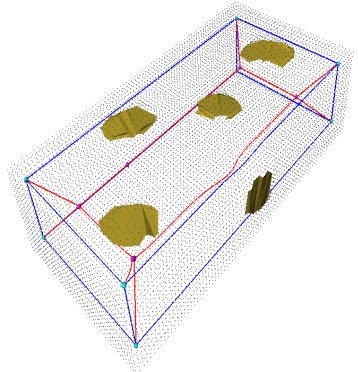

(i)

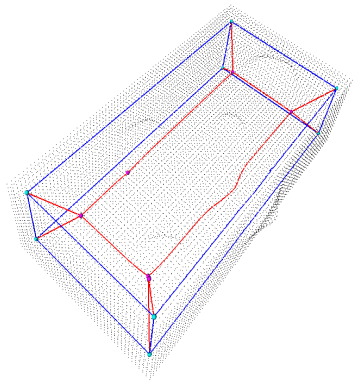

Figure 6: (a) Illustration in 2D of the process of replacing an $A_{1} A_{3}$-I "protrusion" by a circular arc completion between contact points of the $A_{1}^{3}$ circle. (b) In $3 \mathrm{D}$, an $A_{1}^{3}$ and $A_{3}$ curves joined at a pair of $A_{1} A_{3}$ vertices form a loop around the sheet which corresponds to the protrusion. (c) Illustration in $3 \mathrm{D}$ of the process of replacing a "protrusion" by a series of circular arc completions between contact points, indicated by V-shaped hinges (black) linking it to the $A_{1}^{3}$ curve (red), generating a smooth surface patch (golden) interpolating and cutting-off the "bump" above it (d). (e) Example of identified structures for 2 bumps deforming a pair of flat surfaces. (f) Example of a rectangular box uniformly sampled, but deformed by 5 protrusions ( 4 on top, 1 on a side). ( $g$ ) Shock scaffold left after surface meshing (shown in (f)) has been performed, with the three types of transitions due to surface perturbations; in (h) are shown the associated "cut off" patches. (i) Regularized shock scaffold after transition removal. 
(a)

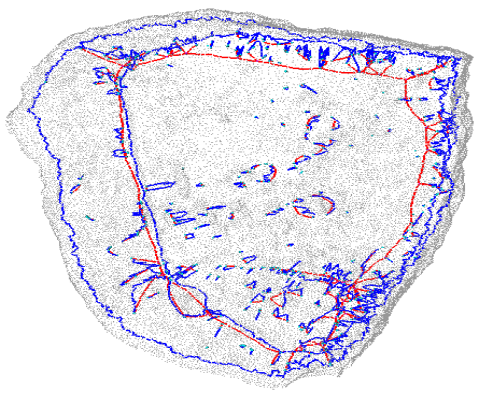

(b)

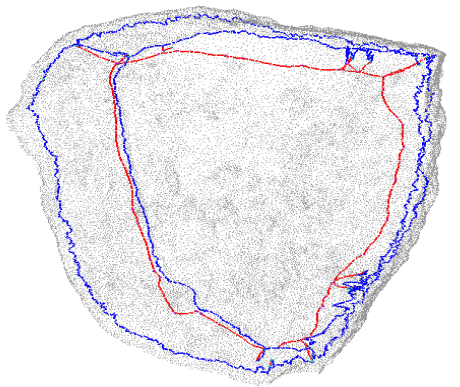

(c)

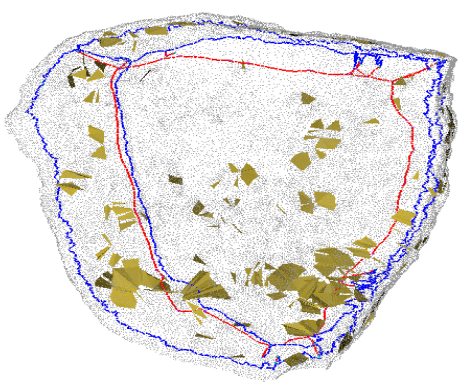

Figure 7: Pot sherd of Fig.1.(a) and its scaffold before (a) and after $(b)$ transition removal; $(c)$ associated "cut off" patches.

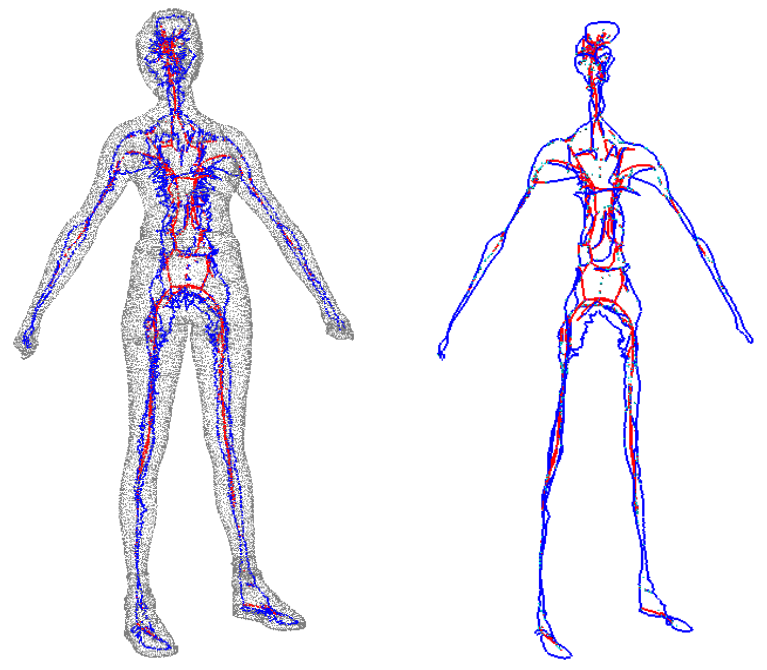

Figure 8: Female body data from Cyberware Inc. (30,432 point generators) and its regularized shock scaffold. (a)

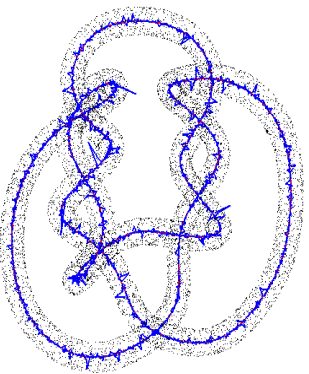

(c)

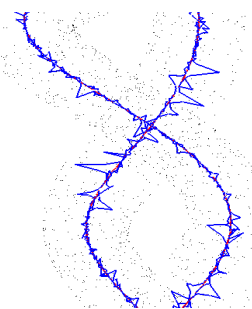

(b)

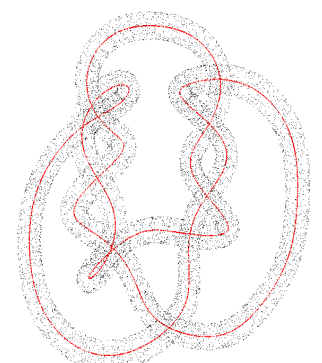

(d)

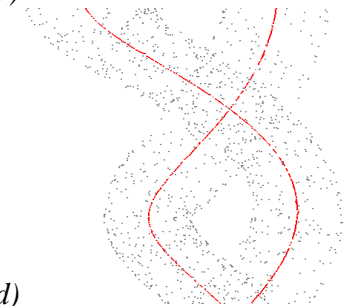

Figure 9: Knot data: 10,000 point generators randomly sampled. (a) and (b) Shock scaffolds before and after transition removal. $(c)$ and $(d)$ : zoom on the scaffolds in $(a)$ and $(b)$.

[3] P. J. Giblin and B. B. Kimia. On the local form of symmetry sets, and medial axes, and shocks in $3 \mathrm{D}$. In $C V P R$, pages 566-573, 2000.

[4] P. J. Giblin and B. B. Kimia. Transitions of the 3D medial axis. In ECCV, volume 2, pages 718-724, 2002.

[5] G.Malandain and S.Fernandez-Vidal. Euclidean skeletons. Image and Vision Computing, 16(5):317-327, 1998.

[6] B. B. Kimia and K. Siddiqi. Geometric heat equation and nonlinear diffusion of shapes and images. CVGIP:IU, 64(3):305-322, 1996.

[7] F. F. Leymarie. 3D Shape Representation via Shock Flows. $\mathrm{PhD}$ thesis, Brown University, 2003.

[8] F. F. Leymarie and B. B. Kimia. Computation of the shock scaffold for unorganized point clouds in 3D. In CVPR, volume 1, pages 821-827, 2003.

[9] F. Mokhtarian and A. Mackworth. Scale-based description of planar curves. IEEE Trans. PAMI, 14(8):789-805, 1986.

[10] E. Pauwels et al. Enhancement of planar shape through optimisation. IEEE Trans. on PAMI, 17:1101-1105, 1995.

[11] P. Perona and J. Malik. Scale-space and edge detection. IEEE Trans. on PAMI, 12(7):629-639, July 1990.

[12] J. Serra. Image Analysis and Mathematical Morphology, volume 1. Academic Press, London, UK, 1982.

[13] D. Shaked and A. M. Bruckstein. Pruning medial axes. Comp. Vision and Image Understand., 69:156-169, 1998.

[14] K. Siddiqi and B. B. Kimia. A shock grammar for recognition. In CVPR, pages 507-513, 1996.

[15] H. Tek and B. B. Kimia. Boundary smoothing via symmetry transforms. JMIV, 14(3):211-223, May 2001.

[16] A. Verroust and F. Lazarus. Extracting skeletal curves from 3D scattered data. The Visual Computer, 16(1):1525,2000 . 